\title{
DESIGN OF A LOW COST DIODE-LASER-BASED HIGH SPECTRAL RESOLUTION LIDAR (HSRL)
}

\author{
Matthew Hayman ${ }^{1}$, Scott Spuler ${ }^{1}$, Bruce Morley ${ }^{1}$, Edwin W. Eloranta ${ }^{2}$ \\ ${ }^{1}$ Earth Observing Laboratory, National Center for Atmospheric Research, PO Box 3000, Boulder, CO, 80307, USA, \\ mhayman@ucar.edu \\ ${ }^{2}$ Space Science and Engineering Center, University of Wisconsin-Madison, 1225 W. Dayton St., Madison, WI, 53706, \\ USA
}

\begin{abstract}
A concept for an eye-safe, semiconductor-based high spectral resolution lidar has been developed at the National Center for Atmospheric Research. The lidar operates at a wavelength of $780 \mathrm{~nm}$ near several rubidium absorption peaks. A rubidium vapor cell is used to block aerosol backscatter in one channel to provide a molecular backscatter measurement for calculating extinction and backscatter ratio (calibrated backscatter).
\end{abstract}

Laser and optical components around $780 \mathrm{~nm}$ are widely developed due to the large growth in atomic cooling and trapping of rubidium. Thus this instrument can be built largely using mature commercial-off-the-shelf parts. The simulation of the conceptual design shown here uses known commercial products and suggests that such an instrument could be used for quantitative profiling of the lower troposphere.

\section{INTRODUCTION}

Commercially available backscatter lidar (e.g. Vaisala Ceilometer, Sigma Space Micropulse Lidar) are a major source of lidar data used in studies relating to clouds and aerosols. The availability, mobility, cost and ease of use associated with these instruments is often sufficiently attractive for scientific researchers to accept the relatively limited supply of quantitative results provided by such systems. The directly measured data product provided by these lidar is attenuated backscatter, in which separating particle backscatter and atmospheric optical depth present an intractable problem.

By contrast, quantitative optical parameters can be accurately and reliably retrieved using Raman and high spectral resolution lidars. Both techniques rely on measuring a molecular backscatter profile to calibrate aerosol backscatter and infer an extinction profile. Raman lidar relies on the unique vibrational Raman spectra of nitrogen or oxygen molecules to separate molecular backscatter from the total aerosol and molecular backscatter signals [1]. Raman lidars are often appealing for their all-inone ability to simultaneously profile clouds and aerosols as well as other important atmospheric state parameters such as temperature and water vapor. However, Raman scattering is several orders of magnitude weaker than Rayleigh scattering so such systems typically require high power lasers which are both expensive, require regular maintenance, and make it difficult to achieve rou- tine unattended deployment.

High spectral resolution lidar (HSRL) provides a second option for quantitative profiling of aerosols and clouds which can operate at eye-safe intensities. An HSRL measures molecular backscatter by blocking the spectrally narrower aerosol backscatter signal with an optical notch filter. Thus, the molecular channel only sees the spectral wings of the molecular backscatter spectrum.

Originally, Fabry-Pérot etalons were used to perform this spectral filtering [2]. These typically come with some level of complexity, requiring tuning, temperature control, limited optical throughput (where etalon size often limits the lidar's primary aperture or field-of-view), and insufficient optical depth at single passes.

Iodine absorption lines have been used to perform spectral filtering [3]. There are several lines with high optical depth near the common $532 \mathrm{~nm}$ doubled Nd:YAG wavelength. Iodine cells require much less temperature control and have no sensitivity to angle of incidence. However, obtaining reliable, low maintenance narrow band lasers at this wavelength continues to be a challenge. Also the highly visible green light presents problems for unattended operation, where the light may serve as a distraction to overhead aircraft. This is particularly problematic in for night operation and instances where slant transmit angles are needed.

Recent developments in water vapor DIAL technology at Montana State University (MSU) and the National Center for Atmospheric Research (NCAR) have shown that semiconductor lasers can be used to build reliable, eyesafe, low cost water vapor profiling systems[4; 5]. We will show here that similar development of a semiconductor laser HSRL, using rubidium absorption lines for spectral filtering, presents a useful opportunity to perform quantitative microphysical measurements of the atmosphere with a reliable, low cost system. Optical elements such a narrow linewith semiconductor lasers and amplifiers are available as mature commercial off the shelf (COTS) components due to their heavy use in atomic cooling and trapping where often times the requirements on the optical elements are much more stringent than what is needed for an HSRL.

The use of lower power lasers and longer wavelengths means the proposed rubidium HSRL (Rb-HSRL) will have lower performance compared to its existing HSRL 
and Raman lidar counterparts. However, compared to existing low cost commercial systems, the Rb-HSRL would be a great leap forward in providing quantitative optical measurements and present new opportunities for deployment on platforms previously incapable of housing such devices.

\section{SIMULATION}

The simulated design of a Rb-HSRL is a derivative of the current design of water vapor DIAL at NCAR [5]. A distributed Bragg reflector semiconductor laser at $780 \mathrm{~nm}$ is coupled into a tapered semiconductor optical amplifier (TSOA). This amplifies the laser and provides the pulse of $15 \mu J$ over a $900 \mathrm{~ns}$ pulse. The beam is expanded to fill the inner part of the transmit telescope with a radius of $10 \mathrm{~cm}$. The laser repetition rate is dictated by an assumed maximum permissible energy (MPE) of $0.47 \mathrm{~mW} / \mathrm{cm}^{2}$. That is, direct exposure to the laser is within eye-safe limits. The resulting pulse repetition rate is just below $10 \mathrm{kHz}$. This repetition rate limits the maximum altitude of the lidar system to less than $15 \mathrm{~km}$, however solar background levels will result in lower resolvable altitudes during daytime operation.

The construction of the system assumes a transceiver configuration where the aperture stop and therefore the telescope is divided into a transmit and receive portion [5]. This is accomplished by placing a mirror with a hole at a plane conjugate to the telescope primary (See Fig. 1). The transmit laser is passed through the hole, while the received light is reflected off the mirror into the detection path. This provides the stability of a transceiver lidar with higher isolation between transmitter and receiver at the cost of reduced receiver area. The receiver telescope has a diameter of $40 \mathrm{~cm}$ where the inner $20 \mathrm{~cm}$ diameter is dedicated to the transmit laser.

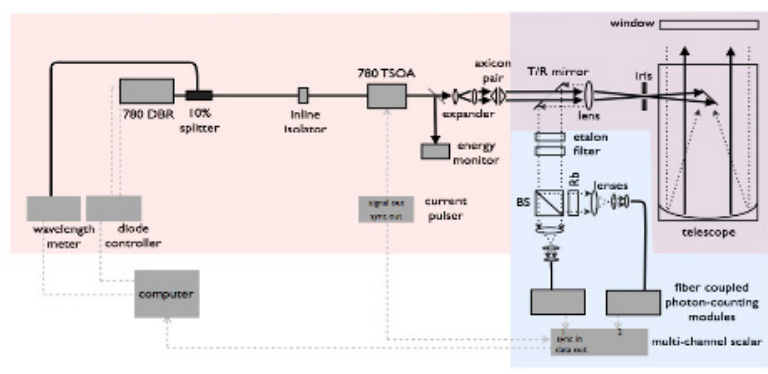

Figure 1: Proposed layout of the Rb-HSRL based on the water vapor DIAL system designed at NCAR/MSU [5]. The instrument uses a shared aperture transceiver for pointing stability with good isolation. A rubidium cell is heated to $320 \mathrm{~K}$ to block aerosol backscatter in the molecular channel.

The receiver system consists of a solid etalon for daylight rejection with a full width half $\max (\mathrm{FWHM})$ of $8 \mathrm{GHz}$ and a free spectral range (FSR) of $50 \mathrm{GHz}$. The light then passes through a 90:10 beam splitter. Because it is generally the weaker signal, $90 \%$ of the received light is directed to the molecular channel through a heated rubidium cell, while the other $10 \%$ is directed onto a detector for the total backscatter measurement. It should be noted that a polarizing beam splitter could easily be installed in the total channel to provide polarization measurements. This comes at the expense of adding another detector and data acquisition channel. It may also require a polarizer in the transmitter.

The Doppler broadened rubidium spectra is shown for naturally occurring isotopic abundance in Fig. 2. In addition, Thorlabs offers an Rb 87 cell which is $98 \%$ pure $(\mathrm{Rb}$ 85 is the other more common isotope of rubidium). For simplicity, we assume in this simulation that we are using the Rb 87 cell provided by Thorlabs ( $7.2 \mathrm{~cm}$ long) as well as their corresponding cell oven. Typically aerosol blocking with optical depth greater than 6 is desirable for accurate liquid cloud characterization. This requires this rubidium cell to be heated to greater than $320 \mathrm{~K}$. The resulting optical depth spectrum is shown in Fig. 2.

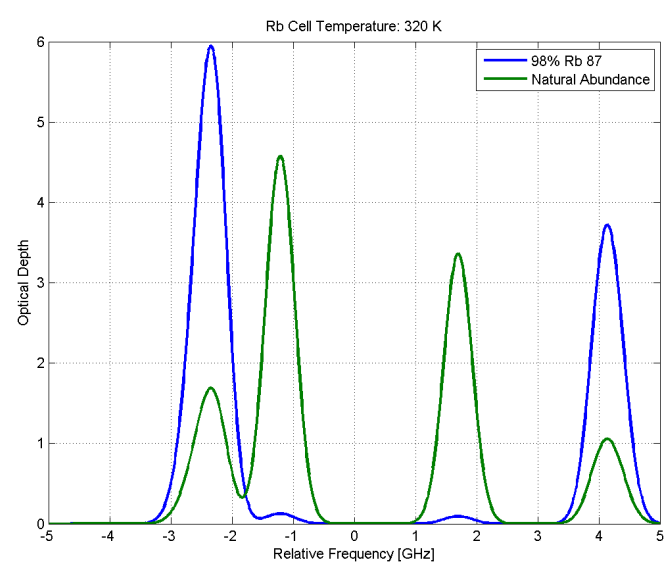

Figure 2: Rubidium absorption spectra for a $7.2 \mathrm{~cm}$ cell at 320 $\mathrm{K}$. The Rb-HSRL design considered here assumes the use of the $\mathrm{Rb} 87$ cell available from Thorlabs.

This simulation uses a U.S. standard atmosphere with aerosol loading chosen to be the lower-decile aerosol backscatter coefficients from page 944, Table 4 in [6], scaled from $10.6 \mathrm{um}$ wavelength to $780 \mathrm{~nm}$. To obtain extinction figures, we assume a constant lidar ratio of 60 . Also an overlap function is roughly approximated where full overlap approximately occurs at

$$
z=\frac{D_{\text {telescope }}}{\theta_{F O V}}
$$

where $D_{\text {telescope }}$ is the telescope diameter and $\theta_{F O V}$ is the full angle field-of-view (FOV) of the receiver.

The profile calculated for the Rb-HSRL was normalized for component, power and wavelength and compared to a similarly normalized profile measured by the NCAR/NSF GV-HSRL. This allows us to better estimate efficiency 
Table 1: Simulation parameters for Rubidium HSRL

\begin{tabular}{ll}
\hline Transmitted pulse width & $900 \mathrm{~ns}$ \\
Transmitted pulse energy & $15 \mu \mathrm{J}$ \\
Pulse repetition rate & $9.8 \mathrm{kHz}$ \\
Laser wavelength & $780 \mathrm{~nm}$ \\
Transmitted beam diameter & $20 \mathrm{~cm}$ \\
Telescope diameter & $40 \mathrm{~cm}$ \\
Effective receiver collection area & $940 \mathrm{~cm}^{2}$ \\
Field-of-view & $150 \mu \mathrm{rad}$ \\
Receiver efficiency & 0.006 \\
Receiver optical bandwidth & $8 \mathrm{GHz}(\mathrm{FWHM})$ \\
Rubidium cell length & $7.2 \mathrm{~cm}$ \\
Rubidium cell temperature & $320 \mathrm{~K}$ \\
Range resolution & $150 \mathrm{~m}$ \\
Solar background & $1.15 \mathrm{mWcm}{ }^{-2} \mu \mathrm{m}^{-1} \mathrm{sr}^{-1}$ \\
\hline
\end{tabular}

terms in the lidar system and develop a conservative performance estimate. In this case, we added a multiple of 0.1 to Rb-HSRL simulations to obtain agreement between the two normalized profiles.

The proposed Rb-HSRL is simulated by calculating all relevant range dependent quantities and applying them to a normalized backscatter spectrum for aerosols (which are assumed to be 1000 times more massive than air molecules), and a temperature and pressure dependent molecular spectrum [7]. The subsequent backscatter spectrum is then multiplied by the relevant receiver filter function (etalon for the total channel, and etalon with rubidium spectra for the molecular channel). The simulation parameters are summarized in Table 1.

The pure backscatter signals are shown in Fig. 3. The course dashed lines indicate the solar background levels and the fine dashed lines indicate the signal level where SNR (signal divided by variance) is 1 . Below an SNR of 1 , integration will not improve the resolution of the signal. Thus, for this particular simulation, the maximum resolvable altitude is approximately $6 \mathrm{~km}$. Cloud backscatter information is still likely to be available at higher altitudes. While not enough to provide full tropospheric profiles, this still represents an promising development where the Rb-HSRL can provide quantitative backscatter data over approximately half of the troposphere. The extinction resolution is shown in Fig. 4. The resolution of the system is too course to resolve aerosols but may be useful for estimating cloud extinction.

Additional improvements to Rb-HSRL may be possible as technology continues to advance. For example, higher energy tapered semi-conductor amplifiers may become available as fabrication of longer chips becomes possible. These would increase the maximum altitude of the system and reduce integration times.

\section{CONCLUSION}

We have demonstrated that a diode based, eye safe HSRL is feasible for making calibrated backscatter measure-

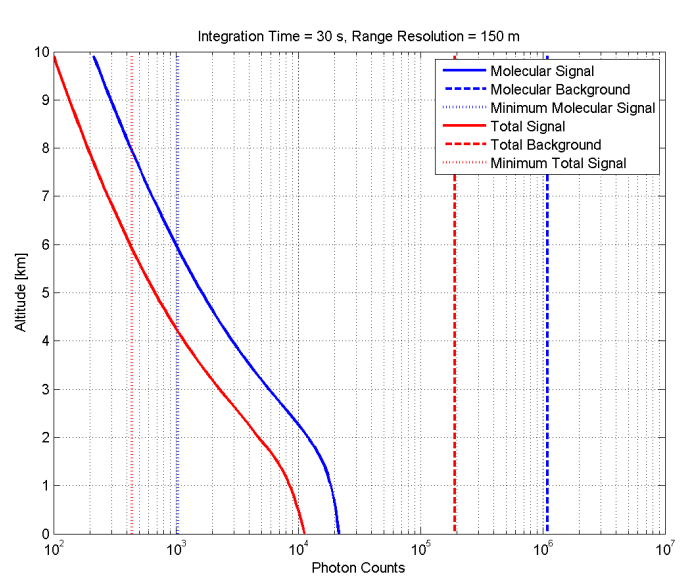

Figure 3: Backscatter (solid), solar background (course dashed) and noise floor (fine dashed) levels of the total (red) and molecular (blue) channels of the Rb-HSRL for a U.S. standard atmosphere [6] with 30 seconds of integration.

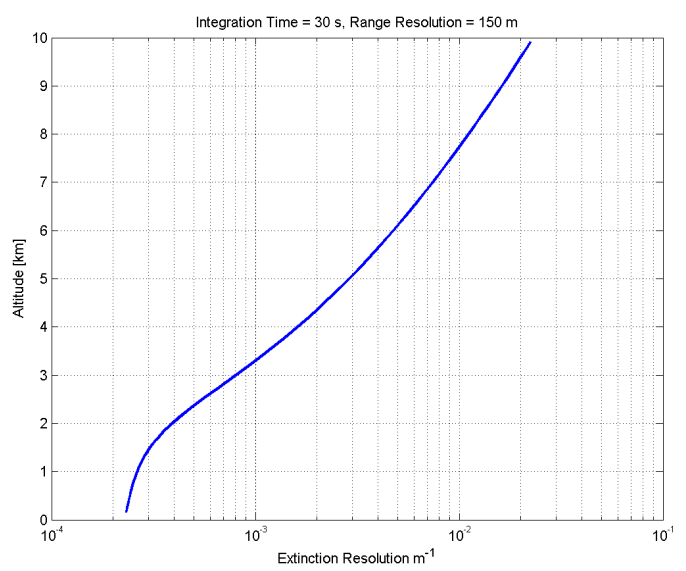

Figure 4: Extinction resolution of the Rb-HSRL for a U.S. standard atmosphere [6].

ments. The system relies on existing commercial components that have largely been proven in the NCAR/MSU water vapor DIAL. It uses a heated rubidium cell as a spectral filter to block aerosol returns. The instrument could retrieve backscatter ratio measurements up to approximately $6 \mathrm{~km}$ during daytime operation. Daytime extinction measurements would largely be limited to clouds. This instrument offers a unique pathway forward in cloud and aerosol profiling due to its potential for unattended operation and low cost.

\section{ACKNOWLEDGMENTS}

The National Center for Atmospheric Research is sponsored by the National Science Foundation. 


\section{REFERENCES}

1. Whiteman, D. N., "Examination of the traditional Raman lidar technique. i. evaluating the temperaturedependent lidar equation," Appl. Opt., 42(15), pp. 2571-2592 (2003).

2. Eloranta, E., Chapter 5: High Spectral Resolution Lidar in Lidar: Range-Resolved Optical Remote Sensing of the Atmosphere, Springer, New York, U.S.A. (2005).

3. Piironen, P., Eloranta, E., "Deomonstration of a highspectral-resolution lidar based on an iodine absorption filter," Opt. Lett. 19, 234-236 (1994).

4. Nehrir, A. R., Repasky, K. S., Carlsten, J. L., Obland, M. D. and Shaw, J. A., "Water vapor profiling using a widely tunable, amplified diode-laser-based differential absorption lidar (DIAL), J. Atmos. Ocean. Technol., 26, pp. 733-745 (2009).

5. Spuler, S. M., Repasky, K. S., Morley, B., Moen, D., Hayman, M., Nehrir, A. R., "Field-deployable diodelaser-based differential absorption lidar (DIAL) for profiling water vapor," Atmps. Meas. Tech, 8, pp. 1073-1087 (2015).

6. Pike, E. R., Sabatier, P.C., Scattering, Two-Volume Set: Scattering and inverse scattering in Pure and Applied Science, Elsevier Science, 2001)

7. Boley, C. D., Desai, R. C., Tenti, G., "Kinetic models and brillouin scattering in a molecular gas," Canadian Journal of Physics, 50(18), pp. 2158-2173, 1972. 